\title{
Parsonage-Turner posterior a vacunación antirrábica por mordedura de murciélago: comunicación de un caso
}

\author{
Parsonage-turner after rabies vaccination due to bat bite: \\ case report
}

\author{
L. Arce Gálvez, J. F. Cantor González y L. M. Rodríguez Vélez
}

Departamento de Medicina Física y Rehabilitación. Universidad del Valle. Cali, Colombia

\section{RESUMEN}

Introducción: Paciente adulto masculino que consulta por mordedura de murciélago. Se realiza aplicación de vacuna antirrábica y toxoide tetánico, tras lo cual presenta alteraciones motoras, sensitivas y dolor de difícil manejo en el miembro superior derecho. Siendo un reto diagnóstico de manera inicial, posterior a estudios electrofisiológicos se consideró un síndrome de Parsonage-Turner. Este caso es relevante por la asociación de vacuna antirrábica no descrita en antecedentes de inmunización en revisiones previas.

Caso clínico: Se le realizaron varias pruebas diagnósticas e imágenes como ecografías, resonancia magnética y estudios electrofisiológicos los cuales confirmaron el diagnóstico de neuritis braquial (síndrome de Parsonage-Turner]. El paciente recibió intervenciones quirúrgicas con fascitomías y neurólisis, además de múltiples manejos farmacológicos para dolor, incluyendo opioides fuertes, neuromoduladores, antidepresivos y medicamentos por bomba intratecal, con disminución solo del $50 \%$ de los síntomas de dolor y afectación a su calidad de vida.

Conclusión: El caso nos muestra cómo el síndrome de Parsonage-Turner, una enfermedad neurológica con etiología desconocida, se puede cronificar en un síndrome doloroso al no tener un diagnóstico temprano o someter al paciente procedimientos no indicados. Este síndrome debe considerarse en la atención de urgencias y consulta externa por sus consecuencias a largo

\section{ABSTRACT}

Introduction: Adult male patient who suffers a bat bite, after which rabies vaccine and tetanus toxoid are administered; later, he presents on the right upper limb severe motor, sensory and pain disorders. Initially a diagnostic challenge, after electrophysiological studies, Parsonage-Turner syndrome was considered. This case is relevant since the association between rabies vaccine and this syndrome has not been described.

Clinical case: Several diagnostic tests and images were performed, including ultrasound, magnetic resonance and electrophysiological studies, which confirmed the diagnosis of brachial neuritis (Parsonage-Turner syndrome). Even though the patient received surgical interventions with fasciotomies and neurolysis as well as multiple pharmacological pain management with strong opioids, neuromodulators, antidepressants and intrathecal pump medications, there was a $50 \%$ decrease in pain symptoms and an impairment of their quality of life.

Conclusion: The case shows a Parsonage-Turner syndrome, a neurological disease with unknown etiology, with difficult diagnosis which can lead to chronic pain syndrome or unnecessary surgical procedures. This syndrome should be considered in emergency care and outpatient care due to its long-term consequences and the difficult management of chronic symptoms. There is a causal relationship reported in the literature with tetanus toxoid, but it was applied prior to symptoms;
Arce Gálvez L, Cantor González JF, Rodríguez Vélez LM. ParsonageTurner posterior a vacunación antirrábica por mordedura de murciélago: comunicación de un caso. Rev Soc Esp Dolor. 2021;28(1):57-61
Recibido: 01-07-2020

Aceptado: 03-03-2021

Correspondencia: Leonardo Arce Gálvez

leonardo.arce@correounivalle.edu.co 
plazo y el difícil manejo de los síntomas crónicos. Hay una relación causal reportada en la literatura con el toxoide tetánico, pero este se aplicó previo a los síntomas; no hay referencias de inicio de síntomas posterior a la vacuna antirrábica, lo cual puede generar a futuro, una relación causal si se encontraran nuevos casos.

Palabras clave: Parsonage-Turner, plexopatía braquial, vacunación, dolor, murciélago, vacuna antirrábica. there are no reports of onset of symptoms after the rabies vaccine, which may generate a causal relationship in the future if new cases are found.

Key words: Parsonage-Turner, brachial plexopathy, vaccination, pain, bat, rabies vaccine.

\section{¿QUÉ SABEMOS ACERCA DE ESTE PROBLEMA?}

- Se ha descrito el síndrome de Parsonage-Turner en inmunización, patologías virales, traumáticas, con una proporción discretamente mayor en hombres.

- La evolución depende del compromiso del plexo braquial y la evolución del dolor en intensidad y tiempo.

- La patología es autolimitada en muchos casos, en los pacientes con complicaciones crónicas tiene grandes implicaciones funcionales y en calidad de vida.

\section{¿QUÉ APORTA ESTE ESTUDIO DE NUEVO?}

- La inmunización inicial con toxoide tetánico y la aplicación de vacuna antirrábica previo a los síntomas del síndrome de Parsonage-Turner, además de la descripción de complicaciones a largo plazo con limitada recuperación secundario a compromiso nervioso difuso e identificación clínica tardía.

\section{INTRODUCCIÓN}

Este caso nos presenta un paciente procedente del suroccidente colombiano, que recibe cuatro dosis de vacuna antirrábica y toxoide tetánico por la mordedura de un murciélago, desarrollando posterior a la aplicación de la última dosis vacuna antirrábica alteraciones motoras, sensitivas y un cuadro de dolor de difícil manejo, cumpliendo características de sindrome de Parsonage-Turner (SPT) (1) (Tabla I). En este síndrome, hasta el $15 \%$ de los casos se presentan posterior a la inmunización para tétanos y tifo [ㄹ] , siendo recurrente en el $26 \%$ de los pacientes en seguimientos de 6 años (3)]. El paciente recibió valoraciones multidisciplinarias en urgencias, hospitalización y consulta externa, por médicos especialistas en ortopedia, fisiatría, neurología, infectología, toxicología, medicina del dolor y manejos intervencionistas por síntomas iniciales y control de dolor con solo mejoría parcial de los mismos.

El paciente cursó clínicamente con signos y síntomas de mal pronóstico para este síndrome: una intensidad alta y duración prolongada del dolor, una afectación difusa del plexo braquial y nervios inferiores (4). Pre-

TABLA |

CARACTERÍSTICAS CLÍNICAS DEL SÍNDROME DE PARSONAGE-TURNER

\begin{tabular}{|c|c|}
\hline & Características del Síndrome de Parsonage-Turner \\
\hline Etiología & $\begin{array}{l}\text { - Desconocida } \\
\text { - Incidencia 1,6/100.000 hab EE.UU (1) } \\
\text { - Predominio masculino 11,5:1 }\end{array}$ \\
\hline Clínica & $\begin{array}{l}\text { - Dolor localizado en hombro y región proximal miembro superior } \\
\text { - Debilidad y atrofia muscular } \\
\text { - Síntomas sensitivos 48-72 \% de pacientes (1] } \\
\text { - Hipoestesias y parestesias } \\
\text { - Síntomas autonómicos }\end{array}$ \\
\hline Evolución & $\begin{array}{l}\text { - Recuperación entre 1-22 meses, } 17 \% \text { con parálisis moderada o severa a los } 3 \\
\text { años, } 1 / 3 \text { de los pacientes con dolor crónico a alteración funcional (1) }\end{array}$ \\
\hline $\begin{array}{l}\text { Electromiografia y } \\
\text { neuroconducción } \\
\text { (electrofisiología) }\end{array}$ & $\begin{array}{l}\text { - Neuroconducción con amplitud reducida, latencia distal prolongada o bloqueo de } \\
\text { conducción. Electromiografía denervación con fibrilaciones, ondas positivas, disminución } \\
\text { en reclutamiento, potenciales de unidad motora polifásicos en reinervación (1] }\end{array}$ \\
\hline
\end{tabular}


sentó cambios autonómicos e inflamatorios locales que llevaron a pensar de manera inicial en complicación infecciosa, que llevaron a realización de procedimientos quirúrgicos y sospecha de un síndrome doloroso regional complejo. Estos síntomas pueden presentarse en el SPT de manera infrecuente $[3,5]$.

\section{CASO CLÍNICO}

\section{Información del paciente}

Hombre de 29 años, natural y procedente de la ciudad de Santiago de Cali, Colombia, que inicia con dolor intenso de características neuropáticas en región proximal del brazo derecho (lugar de vacunación), asociado a hipoestesias, ardor, prurito y calor local, pérdida progresiva de la fuerza, cambios tróficos de la piel, caída de vello y edema; además, dolor en músculos maseteros y de la musculatura facial derecha. Fuera del evento desencadenante el paciente presentaba alergia a tramadol y dipirona, sin otros antecedentes familiares, quirúrgicos, psicológicos o patológicos de interés.

\section{Hallazgos clínicos}

Se encontró paciente con edema en toda la extremidad, de predominio en dorso de la mano y en región supratroclear derecha, con herida en piel por mordedura a nivel de brazo izquierdo sin signos de sobreinfección. Desde el punto de vista neurológico, se encontró debilidad de predominio proximal en la extremidad, reflejos osteotendinosos normales e hipoestesias en el cuarto y quinto dedo. Adicionalmente se observó limitación de la extensión de los dedos, con cambios de la coloración de la piel.

Podemos encontrar la línea de tiempo en la Figura 1.

\section{Evaluación diagnóstica}

Se puede observar en la línea del tiempo de los procedimientos diagnósticos realizados al paciente en miembro superior derecho (Figura 1) que incluyen: ecografía-doppler arterial y venosa sin compromiso vascular; resonancia magnética de toda la extremidad superior derecha con cambios a nivel de la grasa celular subcutánea con edema y trazos de fibrosis en zonas

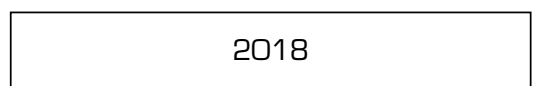

- Mordedura de murciélago

- Aplicación de vacuna antirrábica días $0,3,7,14$. Toxoide tetánico y ATB profiláctico oral

- Inicio de los síntomas

- Doppler arterial normal, doppler venoso sin TVP, cambios inflamatorios grasa del tejido celular subcutáneo

- Ecografía de tejidos blandos: edema distal en codo, sin lesiones vasculares, sin colecciones

- Toxicología: reaccion postvacunal, con plexitis braquial ipsilateral, reacción adversa tipo $B$

- Infectología: colección 18 cc, drenaje quirúrgico, cefepime + daptomicina. Posterior cultivo negativo

- Ortopedia: síndrome compartimental, exploración quirúgica sin colecciones

- Neurología: posible plexitis inflamatoria Parsonage- Turner $10 \%$ de casos puede ser postvacunal

- Clínica de dolor: dolor neuropático, inicia pregabalina 300 mg/día

- MinSalud y secretaría departamental: verificacion de condiciones básicas y garantía de calidad de vacunas

- Clínica del dolor: bloqueo simpático t2-t3 derecho

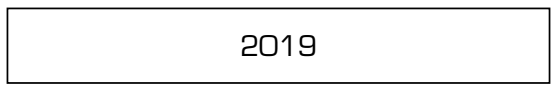

- RM brazo derecho: alteración de grasa celular subcutánea, edema y fibrosis

- Gangliolisis de V par por radiofrecuencia. Metadona, imipramina y oxicodona por medicina del dolor

- Descartada patología reumatológica

- Patología de muestras de brazo con resporte de proceso inflamatorio agudo

- RM de codo derecho signos de miositis edema de tejidos blandos

- RM de antebrazo y muñeca edema de tejidos blandos, sin colecciones 0 abscesos

- Dx síndrome doloroso regional complejo, equimosis y edema

- EMG + NC: lesión axonal y desmielinizante incompleta de plexo braquial de localización difusa con signos de reinervación

- Dx plexitis braquial, síndrome de Parsonage-Turner

- Dx síndrome doloroso regional complejo tipo 2, neuroestimulación percutánea de cordones posteriores

- Inicio de duloxetina por psiquiatría, ideación suicida no estructurada inicio de escitalopram

- Ajuste de medicación a metadona, pregabalina, clonazepam, imipramina

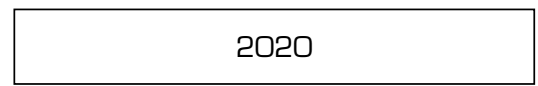

- Falla terapéutica de neuroestimulación, propuesta de bomba intratecal

- Implante intratecal con respuesta bizarra, inicio de alprazolam y fluoxetina

- Retiro implante y bomba de morfina por emesis, náuseas y aumento de dolor

- Inicio de dimenhidrinato por mareo

- Dx de vértigo periférico

- Audiometría; hipoacusia neurosensorial leve bilateral, $100 \%$ discriminación de lenguaje

- Inicio de amitriptilina en la noche

Fig. 1. Línea del tiempo. 
del paquete vasculonervioso compatibles con proceso inflamatorio. El estudio electrofisiológico reporta lesión axonal y desmielinizante incompleta de plexo braquial de localización difusa con signos de reinervación, toxicología considera que el paciente puede estar cursando con una plexitis braquial como reacción adversa tipo $B$ postvacunal; posteriormente rehabilitación y neurología coinciden en que el paciente presenta un posible SPT, que puede aparecer como reacción postvacunal hasta en el $15 \%$ [?] , teniendo en cuenta para el diagnóstico el desarrollo clínico, el tiempo de la vacunación e inicio de síntomas, la localización del dolor, cambios inflamatorios, compromiso funcional y las características del estudio electrofisiológico realizado por el especialista en rehabilitación.

\section{Intervención terapéutica y seguimiento}

Inicialmente, dado el cuadro postvacunal del paciente, se verificó por las autoridades competentes las adecuadas condiciones básicas, la calidad y el procedimiento de aplicación de las vacunas. Dados los cambios inflamatorios presentados de manera inicial, fue sometido a fascitomías y liberaciones de nervio cubital y mediano, e inicio de AINE tipo naproxeno $250 \mathrm{mg}$ cada 8 horas, sin mejoría de síntomas. Posteriormente se modificó el tratamiento analgésico por psiquiatría y medicina del dolor a escitalopram $10 \mathrm{mg}$ día, fluoxetina $20 \mathrm{mg}$ cada 12 horas, alprazolam 0,25 mg cada 12 horas, trazodona $100 \mathrm{mg}$ noche, clonazepam 5 gotas cada 12 horas y amitriptilina 50 mg/día. Adicionalmente, rehabilitación añadió pregabalina $150 \mathrm{mg}$ cada 6 horas y medicina del dolor opioides de tipo oxicodona $60 \mathrm{mg} /$ día, metadona $10 \mathrm{mg}$ cada 8 horas, y capsaicina tópica cada 12 horas. A pesar del uso de dosis máximas, se han ido suspendiendo de manera paulatina al no encontrar una mejoría clínica. Se realizó un intento de control de dolor con neuroestimulador en cordones posteriores por vía percutánea, el cual presenta falla terapéutica con posterior instalación de una bomba intratecal de morfina sin respuesta terapéutica al dolor y efectos indeseados por el paciente. No se consideró administrar otra opción por negativa del paciente ante los síntomas y la no disponibilidad de ziconotide, con lo que fracasaron las opciones intervencionistas. En el momento es manejado con metadona, amitriptilina, pregabalina, fluoxetina y alprazolam, con referencia de control del $40-50 \%$ de los síntomas dolorosos, agudización periódica, adicional dimenhidrinato por otorrinolaringología por cuadro de vértigo posterior a bomba intratecal.

\section{DISCUSIÓN}

Este caso clínico cumple con los lineamientos de aplicación de vacuna de exposición rábica del Ministerio de Salud de Colombia, sin aplicación de suero y con verificación de condiciones técnicas [6]. Se realizó resonancia magnética y electromiografía-neuroconducción como parte del protocolo diagnóstico elegido por personal médico y la toma de ecografía-doppler para descartar compromiso vascular. La intervención multi- disciplinaria y el plan terapéutico de rehabilitación están acordes a las guías de manejo.

No se encontró un hallazgo objetivo documentado de la presencia de compromiso infeccioso, con cultivos negativos e imágenes inespecíficas; sin embargo, el paciente recibió manejo antibiótico de amplio espectro e intervenciones quirúrgicas para lavado y desbridamiento. Estos hallazgos inflamatorios pueden estar acorde a la evolución normal de la enfermedad $(3,5)$. En el manejo del dolor neuropático se utilizaron todas las líneas de manejo descritas (ㄱ), pero se pudo usar lidocaína y gabapentina antes del inicio de opioides fuertes.

EI SPT es un trastorno raro que puede presentarse en todos los grupos etarios, siendo más frecuente en la tercera y séptima década (ㅇ]); con antecedentes relacionados infecciosos, inmunológicos y traumáticos, afectando a cualquier nervio periférico con mayor frecuencia la parte superior del plexo braquial y peor pronóstico en la afectación de la parte inferior $(\underline{4,9})$, sugiriendo además que la fisiopatología implica la predisposición genética, la vulnerabilidad mecánica y el desencadenante inmunológico $(1,4)$. Los estudios de imágenes y electrofisiológicos del paciente se acercan a los reportados en revisiones previas (10). En la mayoría de los casos una recuperación en la debilidad y dolor se da en el primer mes posterior al inicio de los síntomas teniendo al $75 \%$ [의 de los casos, con recuperación completa a los dos años y al $90 \%$ a los 3 años, pero pudiendo presentar una recurrencia mayor al $20 \%$ de los paciente en los siguientes 6 años (프); en este caso con una complicación de dolor exacerbada por los múltiples procedimientos quirúrgicos además de la pérdida funcional y discapacidad, la vacunación con toxoide tetánico y vacuna antirrábica está presente en este caso, siendo la vacunación de toxoide tetánico un antecedente ya descrito en diferentes reportes (3]), pero con la novedad de inicio de síntomas posterior a la aplicación de la última dosis de vacuna antirrábica, siendo un factor adicional en este paciente.

Se considera en futuros casos incluir el SPT como diagnóstico diferencial en los pacientes con clínica de debilidad y dolor posterior a vacunación, intervenciones y demás factores asociados como infecciones virales, ejercicio de alta intensidad, puerperio, perioperatorio y enfermedades del tejido conectivo como antecedente, reconocer los síntomas locales que pueden estar presentes que no median patología infecciosa, además del correcto diagnóstico con el uso de imágenes, electromiografía y neuroconducción de la extremidad afectada; el inicio temprano de medicación y plan de rehabilitación como pilar de la recuperación del paciente con la ganancia y mantenimiento de rangos de movilidad articular la prevención de contracturas, el fortalecimiento muscular además del adecuado manejo del dolor con la posibilidad de inicio de esteroides de manera temprana pueden dar un pronóstico más favorable de recuperación, teniendo en cuenta que no hay un tratamiento específico descrito para SPT.

\section{CONCLUSIÓN}

EI SPT puede estar asociado a múltiples antecedentes inmunológicos, incluida la vacuna antirrábica, la 
cual se debe considerar y describir con claridad en los antecedentes de cualquier paciente con SPT. Es vital el diagnóstico adecuado en fases iniciales para evitar las intervenciones y complicaciones a largo plazo, además de una intervención en dolor y rehabilitación dirigida por la evidencia de manejo actual y un objetivo funcional claro.

\section{AGRADECIMIENTOS}

Al del departamento de Medicina Física y Rehabilitación de la Universidad del Valle y a la Dra. María Paz Grisales Gafaro.

\section{CONFLICTO DE INTERESES}

Ninguno.

\section{FINANCIAMIENTO}

Los autores no recibieron financiamiento para llevar a cabo este estudio.

\section{BIBLIOGRAFÍA}

1. Smith CC, Bevelaqua AC. Challenging pain syndromes: Parsonage-Turner syndrome. Phys Med Rehabil Clin. 2014;25(2):265-77. DOI: 10.1016/j.pmr.2014.01.001.
2. Parsonage MJ, Turner JW. Neuralgic amyotrophy: shoulder girdle syndrome. Lancet. 1948;1(6513):973-8. DOI: 10.1016/s0140-6736(48)90611-4

3. Van Alfen N, Van Engelen BG. The clinical spectrum of neuralgic amyotrophy in 246 cases. Brain. 2006; 29(2):438-50. DOI: 10.1093/brain/awh722.

4. Procopio FB, Montero SAR. Síndrome de ParsonageTurner. Revisión bibliográfica. Semin Fund Esp Reumatol. 2010;11(4):144-51. DOl: 10.1016/j.semreu.2010.05.002.

5. Stutz CM. Neuralgic amyotrophy: Parsonage-Turner Syndrome. Journal of Hand Surgery. 2010;35(12):2104-6. DOI: 10.1016/j.jhsa.2010.09.010.

6. Instituto Nacional de Salud Grupo de vigilancia y control de enfermedades transmisibles, 13 de agosto de 2010. Protocolo de vigilancia de rabia [Internet]. Dr. Víctor Hugo Álvarez Subdirector de Vigilancia y Control en Salud Pública. [Citado 2020, Jun 16] Disponible en: www.minsalud.gov.co/Documentos\%20y\%20Publicaciones/Protocolo\%2ORabia.pdf

7. Finnerup NB, Otto M, McQuay HJ, Jensen TS, Sindrup SH. Algorithm for neuropathic pain treatment: an evidence based proposal. Pain. 2005;118(3):289-305. DOI: 10.1016/j. pain.2005.08.013.

8. Tsairis P, Dyck PJ, Mulder DW. Natural history of brachial plexus neuropathy: report on 99 patients. Arch Neurol. 1972;27(2):109-17. DOI: 10.1001/archneur. 1972.00490140013004.

9. Tjoumakaris FP, Anakwenze OA, Kancherla V, Pulos N. Neuralgic amyotrophy (Parsonage-Turner syndrome). J Am Acad Orthopaedic Surg. 2012;20(7):443-9. DOI: 10.5435/ JAAOS-20-07-443.

10. Feinberg JH, Radecki J. Parsonage-turner syndrome. HSS J. 2010;6(2):199-205. DOI: 10.1007/s11420-010-9176-x . 